\title{
EUropean Heliospheric FORecasting Information Asset 2.0
}

\author{
Stefaan Poedts ${ }^{1,12, *}$, Andrea Lani ${ }^{1}$, Camilla Scolini ${ }^{1,2}$, Christine Verbeke ${ }^{1}$, Nicolas Wijsen ${ }^{1}$, Giovanni Lapenta ${ }^{1}$, \\ Brecht Laperre $^{1}$, Dimitrios Millas ${ }^{1}$, Maria Elena Innocenti ${ }^{1}$, Emmanuel Chané ${ }^{1}$, Tinatin Baratashvili ${ }^{1}$, \\ Evangelia Samara ${ }^{1}$, Ronald Van der Linden ${ }^{2}$, Luciano Rodriguez ${ }^{2}$, Petra Vanlommel ${ }^{2}$, Rami Vainio ${ }^{3}$, \\ Alexandr Afanasiev ${ }^{3}$, Emilia Kilpua ${ }^{4}$, Jens Pomoell ${ }^{4}$, Ranadeep Sarkar ${ }^{4}$, Angels Aran ${ }^{5}$, Blai Sanahuja ${ }^{5}$, \\ Josep M. Paredes ${ }^{5}$, Ellen Clarke ${ }^{6}$, Alan Thomson ${ }^{6}$, Alexis Rouilard ${ }^{7}$, Rui F. Pinto ${ }^{7,13}$, Aurélie Marchaudon ${ }^{7}$, \\ Pierre-Louis Blelly ${ }^{7}$, Blandine Gorce ${ }^{7}$, Illya Plotnikov ${ }^{7}$, Athanasis Kouloumvakos ${ }^{7}$, Bernd Heber $^{8}$, \\ Konstantin Herbst ${ }^{8}$, Andrey Kochanov ${ }^{9}$, Joachim Raeder ${ }^{10}$, and Jan Depauw ${ }^{11}$ \\ ${ }^{1}$ KU Leuven, 3000 Leuven, Belgium \\ 2 Royal Observatory of Belgium, 1180 Ukkel, Belgium \\ 3 Department of Physics and Astronomy, University of Turku, FI 20014, Finland \\ ${ }^{4}$ Department of Physics, University of Helsinki, PO Box 64, 00014 Helsinki, Finland \\ ${ }^{5}$ Universitat de Barcelona, 08007 Barcelona, Spain \\ ${ }^{6}$ British Geological Survey, The Lyell Centre, Research Avenue South, Edinburgh EH14 4AP, United Kingdom \\ ${ }^{7}$ Institut de Recherche en Astrophysique et Planétologie (IRAP), CNRS, Université de Toulouse et CNES, 31400 Toulouse, France \\ ${ }^{8}$ Christian-Albrechts-Universität zu Kiel, 24118 Kiel, Germany \\ 9 Andrey Kochanov (Company), 3001 Leuven, Belgium \\ ${ }^{10}$ Space Consulting International LLC (Company), Durham, 03824 NH, USA \\ ${ }^{11}$ Space Applications Services (Company), 1932 Brussel, Belgium \\ ${ }^{12}$ Institute of Physics, University of Maria Curie-Skłodowska, 20-031 Lublin, Poland \\ ${ }^{13}$ LDE3, CEA Saclay, Univsersité Paris-Saclay, Gif-sur-Yvette, France
}

Received 17 July 2020 / Accepted 30 September 2020

\begin{abstract}
Aims: This paper presents a H2020 project aimed at developing an advanced space weather forecasting tool, combining the MagnetoHydroDynamic (MHD) solar wind and coronal mass ejection (CME) evolution modelling with solar energetic particle (SEP) transport and acceleration model(s). The EUHFORIA 2.0 project will address the geoeffectiveness of impacts and mitigation to avoid (part of the) damage, including that of extreme events, related to solar eruptions, solar wind streams, and SEPs, with particular emphasis on its application to forecast geomagnetically induced currents (GICs) and radiation on geospace. Methods: We will apply innovative methods and state-of-the-art numerical techniques to extend the recent heliospheric solar wind and CME propagation model EUHFORIA with two integrated key facilities that are crucial for improving its predictive power and reliability, namely (1) data-driven flux-rope CME models, and (2) physics-based, self-consistent SEP models for the acceleration and transport of particles along and across the magnetic field lines. This involves the novel coupling of advanced space weather models. In addition, after validating the upgraded EUHFORIA/SEP model, it will be coupled to existing models for GICs and atmospheric radiation transport models. This will result in a reliable prediction tool for radiation hazards from SEP events, affecting astronauts, passengers and crew in high-flying aircraft, and the impact of space weather events on power grid infrastructure, telecommunication, and navigation satellites. Finally, this innovative tool will be integrated into both the Virtual Space Weather Modeling Centre (VSWMC, ESA) and the space weather forecasting procedures at the ESA SSCC in Ukkel (Belgium), so that it will be available to the space weather community and effectively used for improved predictions and forecasts of the evolution of CME magnetic structures and their impact on Earth. Results: The results of the first six months of the EU H2020 project are presented here. These concern alternative coronal models, the application of adaptive mesh refinement techniques in the heliospheric part of EUHFORIA, alternative flux-rope CME models, evaluation of data-assimilation based on Karman filtering for the solar wind modelling, and a feasibility study of the integration of SEP models.
\end{abstract}

Keywords: Space weather / CMEs / SEPs

*Corresponding author: Stefaan. Poedts@kuleuven. be 


\section{General description and objective(s)}

\subsection{Aims and motivation}

The EUHFORIA 2.0 project aims at developing an advanced space weather forecasting tool. The project addresses the geoeffectiveness of the impacts of CMEs, CIRs, and SEPs and mitigation of (part of) the damage these cause. It also considers extreme events, but the emphasis is on improving the prediction of "normal" space weather and its effects, in particular on its applications to forecast geomagnetically induced currents (GICs) and radiation on geospace. The project thus addresses many challenging aspects of space weather that are interlinked in a complicated way from Sun to Earth and provides therefore also the potential for some scientific breakthroughs.

Our society is becoming increasingly dependent on technologies and infrastructures that the different space weather phenomena can damage, including power grids, satellites in orbit, and global communication and navigation infrastructures. The ultimate driver of space weather disturbances is the Sun. The most prominent forms of solar activity are coronal mass ejections (CMEs), enormous eruptions of plasma (up to $10^{13}-10^{16} \mathrm{~g}$ ) and magnetic field into interplanetary space at velocities up to several thousand kilometres per second (Webb \& Howard, 2012). When sampled in situ by a spacecraft, they are termed interplanetary CMEs (ICMEs). The background solar wind is bimodal and consists of fast and slow streams, and their compressed interaction regions known as stream interaction regions (SIRs) or co-rotating interaction regions (CIRs) (e.g., Owens \& Forsyth, 2013). Associated with these bulk plasma phenomena are high-energy particle populations known as solar energetic particle (SEP) events (e.g., Lario \& Simnett, 2004), which originate through energisation processes occurring at the site of solar flares and at coronal and interplanetary shocks associated with CMEs, and also with SIRs/CIRs (Fisk \& Lee, 1980). Desai \& Giacalone (2016) state that "Solar energetic particles, or SEPs, from suprathermal (few $\mathrm{keV}$ ) up to relativistic ( $\sim f e w \mathrm{GeV})$ energies are accelerated near the Sun in at least two ways: (1) by magnetic reconnection-driven processes during solar flares resulting in impulsive SEPs, and (2) at fast coronal-mass-ejection-driven shock waves that produce large gradual SEP events".

Direct interactions of CMEs and solar wind streams with the Earth's magnetosphere and SEPs represent two very different chains, both however crucial for space weather. While solar wind, CMEs, and SIRs/CIRs arrive at Earth orbit typically in 1-5 days, high-energy SEPs arrive only in tens of minutes. In contrast to the bulk plasma propagation, SEPs with energies of $\mathrm{keV}-\mathrm{GeV}$ follow trajectories constrained by the interplanetary magnetic field (IMF) orientation. CMEs and SIRs cause disturbances in the geomagnetic field, radiation environment surrounding the Earth (so-called Van Allen Belts) and various current systems in the magnetosphere and ionosphere with effects reaching to the ground.

CMEs are the key drivers of strong and extreme magnetic storms. They are most important at solar maximum, but can cause (extreme) storms at any phase of the solar cycle, including solar minimum (e.g., storm in February 1986; Riley, 2012) and also during weaker solar cycles (e.g., Kilpua et al., 2015;
Liu et al., 2018). CIRs/SIRs, in turn, drive mainly weak to moderate storms, but they effectively enhance electrons to relativistic energies in the radiation belts. SEPs can penetrate the magnetosphere posing a significant threat to satellites. The most energetic SEPs can penetrate even down to the upper atmosphere, where they can have a significant effect on chemistry and result in an atmospheric cascade called a ground level enhancement (GLE). The mutual interaction of CMEs can substantially increase both their potential to accelerate particles, and their geoeffectiveness (e.g., Farrugia et al., 2006). In a "perfect storm" scenario (Liu et al., 2015), the first CME "clears out" the ambient solar wind plasma, such that the subsequent CME will experience a minimal drag and will reach Earth with high speed resulting in major space weather effects throughout the terrestrial system.

Current space weather modelling tools, however, lack several crucial aspects which clearly limits their forecasting capability, namely related to (1) interfacing different models from the Sun to the magnetosphere and ground effects models, (2) predicting in advance the internal magnetic field of Earthimpacting CMEs (this is also a vital aspect to understand and forecast CME-CME interactions), and (3) having capability to predict SEP events.

The information on the solar wind conditions impacting the Earth is currently basically only available at the Lagrangian point L1 from where it takes only about $30 \mathrm{~min}$ to $1 \mathrm{~h}$ to reach our planet, i.e., clearly less than the 1-2 days required by most space weather end users. Most critically, there are no measurements or practical tools to estimate the magnetic field in CMEs before they arrive at the Earth's magnetosphere. Even a fast and strong CME impacting Earth may pass with only minor effects if its magnetic field is directed mainly northward. SEPs and related effects, in turn, are primarily determined by the speed, shape and extent of a CME when it is launched from the Sun, as well as by the properties of the ambient corona the CME surges into. Considering the effects from direct interactions, there should be time to predict and mitigate their geoeffectiveness well in advance as we observe the CME eruption 1-4 days before their arrival at Earth orbit. Although similar lead times cannot be expected for SEPs, which propagate in some tens of minutes from the Sun to the Earth in magnetically well-connected events, accurate modelling can crucially increase our capability to predict the duration and severity of the solar radiation storms that have or are about to commence after western flares and CMEs. For poorly connected eastern events, however, physics-based modelling can significantly improve even the lead time, in particular, if observations from L5 are available, which would allow one to assimilate observations from a better-connected location.

\subsection{Objectives}

EUHFORIA has already been integrated into the ESA Virtual Space Weather Modelling Centre (VSWMC) (Poedts, 2018) and has been coupled to several other models within this framework (see the example visualized in Fig. 1). The VSWMC models are available to the space weather user community via the SWE portal (http://swe.ssa.esa.int/), the main user interface of the ESA SWE network (Poedts et al., 2020).

In the EUHFORIA 2.0 project, we will make several critical improvements to EUHFORIA. Our main focus here is on the 


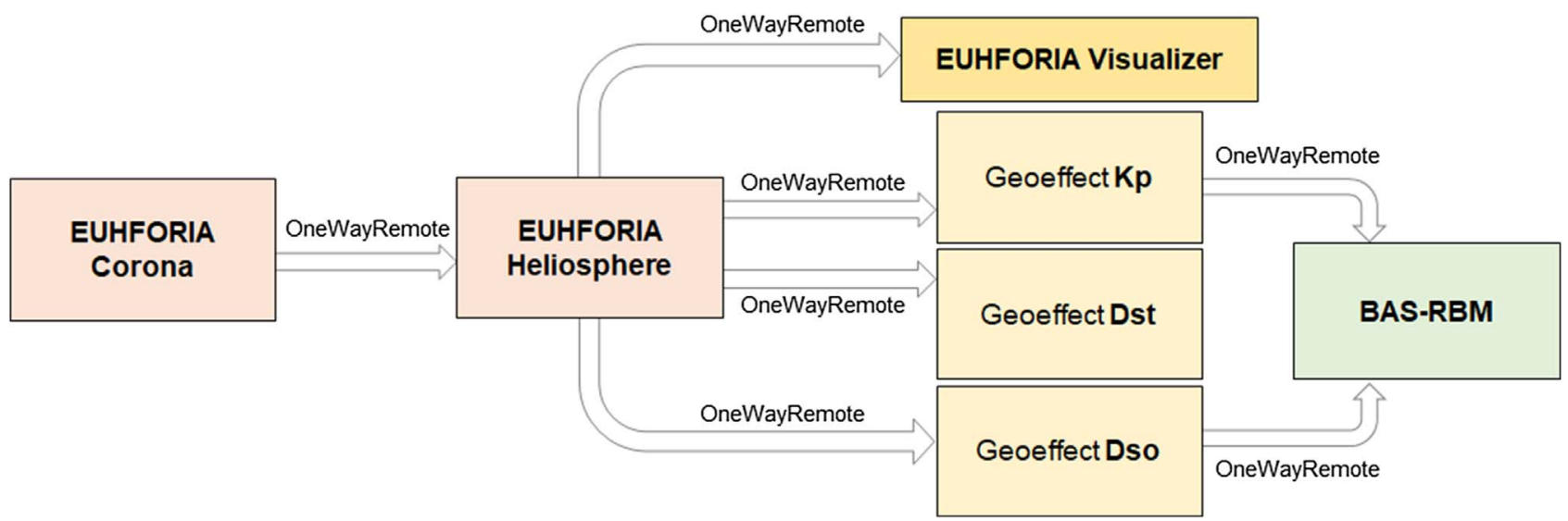

Fig. 1. One of the Sun-to-Earth modelling chains implemented in the VSWMC that became operational in 2019 (see Poedts et al., 2020 ). In this chain EUHFORIA is coupled to models to determine the $K p$ and $D s t$ indices and the plasma sphere stand-off distance, based on the synthetic wind data at L1 from EUHFORIA. The $K p$ index and the plasma sphere stand-off distance (Dso) are then used to drive the British Antarctic Survey Radiation Belt Model.

most urgent physical challenges and damaging impacts that can be mitigated. Thus, the specific objectives are:

Objective 1: To provide accurate predictions of plasma and magnetic field in the near-Earth solar wind by improving our heliospheric wind and CME evolution model EUHFORIA by implementing data-assimilation techniques (using both available and potential L5 and Solar Orbiter data) as well as determine the internal magnetic structure of CMEs using advanced flux-rope models constrained by data-driven and machine learning techniques.

Objective 2: To develop a global coronal Magneto HydroDynamics (MHD) model for EUHFORIA, capable of quantifying the source regions of CMEs and the global coronal magnetic field.

Objective 3: To integrate current state-of-the-art SEP transport models in EUHFORIA for simulation of SEP emission from coronal shocks and to develop methodology and tools for predicting the SEP emission from CMEs.

Objective 4: To develop an operational prediction tool for GICs in power grids.

Objective 5: To develop more reliable operational prediction tools for harsh radiation in geospace.

Objective 6: To exploit EUHFORIA by creating completely novel space weather forecasting service facilities tailored carefully to the needs of selected target groups.

Referring to the modelling chain in Figure 1, we will replace the coronal model in EUHFORIA with a more advanced one, improve the heliospheric part of EUHFORIA (using data assimilation techniques), and couple our SEP transport and acceleration models to EUHFORIA so that we put the SEP source much closer to the Sun and capture the high-energy events too. The concept has been proven already (Wijsen et al., 2019a, b), see below). Moreover, in addition to the geo-indices models mentioned in Figure 1, we will couple a magnetospheric model (OpenGGCM) and GIC and radiation models to EUHFORIA 2.0. This will enable us to replace the nowcasts given by these models to forecasts with up to 5 days' notice.
To maximise the impact, our dissemination and exploitation plan is tailored carefully to the needs of the target groups. The EUHFORIA 2.0 forecast tool will provide reliable quantitative predictions of the space environment parameters at L1 and other satellite positions in the solar system, and forecast GICs in elements of the interconnected European power grid and radiation in the ISS, satellites and public airplanes.

\subsection{Key science questions}

The Key science questions of the EUHFORIA 2.0 are also inspired by COSPAR roadmap recommendations (Schrijver et al., 2015):

1. What is the global coronal field that drives the solar-wind plasma and magnetic field from Sun to Earth and what coronal parameters affect the solar wind at 1 AU the most?

2. How and to what extent do the initial eruption features and the interaction with the solar wind affect (erode, deform) the properties and geoeffectivity of CME-driven IP shocks and ICMEs?

3. How are SEPs produced and transported to 1 AU over the course of CMEs?

4. To what extent does the ambient solar wind play a role in determining whether we observe large SEP events when a big and fast CME event occurs?

5. What are the factors which control the generation of geomagnetically-induced currents (GICs) and of harsh radiation in geospace (involving the coupling of solar wind disturbances to internal magnetospheric processes in the magnetosphere and the ionosphere below)?

\section{CONCEPT and methodology}

\subsection{Project concept}

Interplanetary CMEs (ICMEs) are the main drivers of space weather. Therefore, the modelling of CME onset, SEP emission, 
and their interplanetary propagation up to the impact on the Earth's magnetosphere (affecting the ionosphere, thermosphere, radiation belts, etc.) is pivotal for reliable space weather forecasts. Regional warning centres, e.g. in Brussels (at the Royal Observatory of Belgium), provide daily forecasts using several semi-empirical and simulation models that have been developed for this purpose. There are, however, two major problems related to our current forecasting capabilities. First of all, many of the currently available space weather models are oversimplified, leaving out some key physics, because these are complicated (multi-scale/multi-physics) and/or CPU demanding. The second problem lies in interfacing the different models related to the different domains involved (e.g., the solar corona, the heliospheric solar wind, the CME onset and propagation, SEP events, the terrestrial magnetosphere and ionosphere, etc.) in a consistent coupling framework. Therefore, a SEP prediction model needs to be coupled to the CME propagation and impact model and a comparison between observations and simulation outputs must be carried out to validate any new or upgraded model.

Current CME propagation models, including ENLIL (Odstrcil, 2003) and SUSANOO (Shiota \& Kataoka, 2016), all have limitations: (1) they use a very simplified background solar wind model; (2) they use over-simplified CME models that take at most marginally into account the structure of the magnetic field within the CME itself; (3) they describe the CME early propagation only in a simplistic way or not at all (when introduced only at 0.1 AU like e.g., cone CME models); (4) they do not provide any information about the SEP emission and transport properties generated by solar flares and the CME leading shock fronts; and (5) they are not coupled with magnetospheric/ionospheric and effects models. Recently, first attempts were made to include the internal magnetic structure of the CMEs in ENLIL, in the Space Weather Modelling Framework (Tóth et al., 2005), in SUSANOO (Shiota \& Kataoka, 2016), and in EUHFORIA (Scolini et al., 2019; Verbeke et al., 2019), but none of them are yet used for operational space weather forecasting.

The EUHFORIA project offers an opportunity to build and validate a new advanced space weather forecasting tool, covering both geomagnetic storms from direct interactions of CMEs and other large-scale solar wind structures with the Earth's magnetic environment, and the SEPs generated radiation storms. This builds on the state-of-the-art model EUHFORIA, a 3D MHD solar and heliospheric model that simulates the solar wind and the evolution of a superimposed CME structure from 0.1 AU to $2 \mathrm{AU}$ (i.e. including the orbits of both Earth and Mars) (see Pomoell \& Poedts, 2018; Fig. 2). Wijsen et al. (2019a, b) have already combined EUHFORIA output with a novel SEP transport model solving the focused transport equation with Monte Carlo techniques. At the same time, advanced numerical simulation models have been developed for the acceleration and transport of particles in the corona enabling to get a deeper understanding of the complexity of the interaction between coronal shocks and solar magnetic fields (Afanasiev \& Vainio, 2013; Afanasiev et al., 2014; Vainio et al., 2014, 2015, 2018a, 2018b).

In EUHFORIA, the CMEs are modelled with a magnetic flux-rope, thus taking into account the crucial internal magnetic structure. This enables more reliable CME evolution simulations, taking into account the effects of erosion and deflection (occurring through magnetic reconnection of the internal magnetic field with the magnetic field of the ambient solar wind) and deformation (due to the interaction with the ambient solar wind), and predictions of the geoeffectiveness of an event (which depends largely on the sign and magnitude of the $B z$-component, i.e. perpendicular to the equatorial plane). It has been shown that the use of a spheromak CME model significantly improves the predictions (Scolini et al., 2019, 2020; Verbeke et al., 2019).

As mentioned above, all the current operational heliospheric wind and CME propagation models completely ignore SEP acceleration and transport. Yet, solar energetic particle events can affect communications and airline safety, and affect satellites by radiation damage to electronics. Protons of more than $30 \mathrm{MeV}$ could kill astronauts since these can penetrate spacesuits and spacecraft walls. Hard particle energy spectra can contain large fluxes of hundreds of $\mathrm{MeV}-\mathrm{GeV}$ type superenergetic particles, which can reach low earth orbit (LEO) satellites and even penetrate into the safest areas of spacecraft. The major innovation of the current project will thus be the integration of state-of-the-art SEP transport and emission models into a physics-based and self-consistent model. This will enable to understand, quantify and even forecast the origin and evolution of SEP events.

\subsection{Methodology}

The methodology of the proposed project is directly linked to the six specific objectives mentioned in Section 1.2, namely as follows.

Objective 1: Implementing advanced flux-rope models for the internal structure of CMEs.

We will improve the current wind model in EUHFORIA using data-assimilation techniques exploiting currently available satellite data and exploring the usefulness of L5 data. We will also apply machine learning techniques to quantify the sensibility of the predictions on the CME input parameters in order to optimize the ensemble modelling for the forecasts. We will also explore Lagrangian methods to increase the cost-effectiveness, starting from the SLURM code developed at KU Leuven (Bacchini et al., 2017), and coupling it to EUHFORIA to demonstrate the ability to run a rapid simulation of CMEs.

A CME model should be capable of providing a reasonable 3D geometry fit, include typical deformations (expansion, deflection, rotation, flattening ("pancaking"), skew (due to solar rotation)), and have a 3D internal magnetic field configuration with a low, nearly constant twist. We will implement the Fri3D model (Isavnin, 2016) as well as other flux-rope models.

Objective 2: Developing an improved coronal model for EUHFORIA.

This objective will be tackled by developing novel models of the solar coronal magnetic field and plasma environment and tools to determine realistic initial CME and shock parameters from the low corona up to $0.1 \mathrm{AU}$. We will develop an advanced MHD-based model of the solar corona by extending our current coronal model (Pomoell \& Vainio, 2012). The new model will include a detailed description of coronal thermodynamics, including anisotropic heat conduction, separate ion and electron temperatures and radiative losses. The coronal heat input is provided by an Alfvén wave turbulence model that 

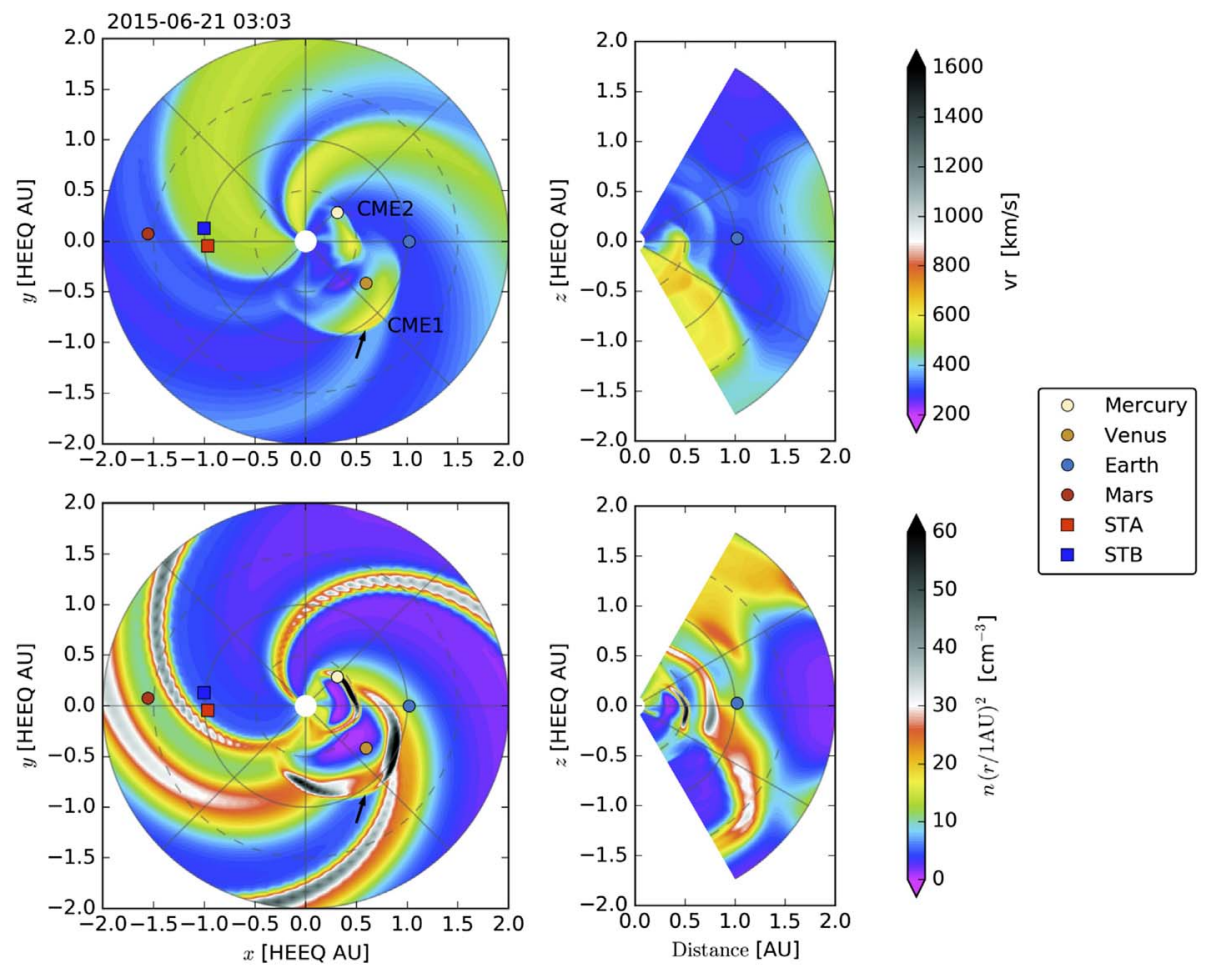

5

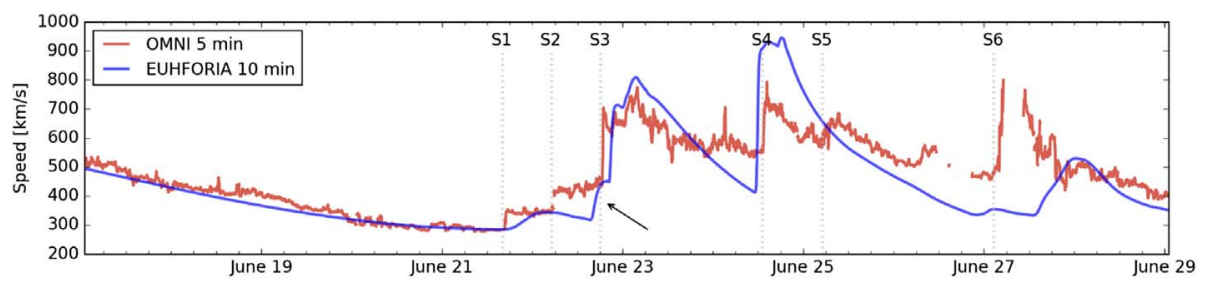

Fig. 2. Above: Snapshot of a EUHFORIA simulation at 03:03 UT on June 21, 2015. Below: radial velocity at L1 as measured (red) and simulated (blue) from (Pomoell \& Poedts, 2018).

has shown to reproduce well the coronal large-scale extreme ultraviolet emission (Van der Holst et al., 2014).

In addition, a 3D coronal shock wave propagation module will be developed to provide quick modelling of shock wave properties in the corona and establish how these shocks connect to specific points of interest in the inner heliosphere. This module will provide the critical shock parameters modelled in 3D which will be used as inputs for the SEP emission modelling.

We will also develop tools for obtaining realistic and practical information of initial CME and shock parameters to constrain the new flux rope models (see Objective 1) and for the SEP forecasting models (see Objective 5). We explore additionally a fully data-driven modelling approach of erupting coronal magnetic fields provided by the supporting UH ERC project SolMAG (PI: Emilia Kilpua) to obtain CME magnetic structure self-consistently and time-dependently without the intervention of the modeller (Pomoell et al., 2019; Price et al., 2019).

Objective 3: Integrating current state-of-the-art SEP transport models in EUHFORIA.

The University of Turku (UTU) team has developed stateof-the-art numerical simulations for particle acceleration at shocks, including the Coronal Shock Acceleration (CSA) simulation model (Vainio \& Laitinen, 2007), which can accommodate global heliospheric field configurations. The more recent model SOLar Particle Acceleration in Coronal Shocks (SOLPACS), uses a physically accurate description of microphysics but is presently limited to local simulation volumes around the shock (Afanasiev et al., 2015). For the downstream side of the shock, the UTU model suite uses a test-particle Monte Carlo simulation called DownStream Propagation Model (DSPM), solving the Parker equation in a prescribed bulk-plasma flow field with a prescribed spatial diffusion tensor.

The UB team in collaboration with KU Leuven team have developed a Shock-and-Particle (SaP) model (Pomoell et al., 2015), which is solving a focused transport equation in a Parker-spiral magnetic field and constant solar wind flow. Unlike CSA/SOLPACS, SaP is not self-consistent in terms of energy exchange with the scattering waves, but its advantage is that the method is computationally efficient, which makes it an attractive alternative for operational modelling.

Moreover, the KU Leuven, University of Barcelona (UB) and University of Helsinki (UH) teams developed the Particle radiation asset directed at interplanetary space exploration model (PARADISE; Wijsen, 2020, see Fig. 3), a Monte Carlo 

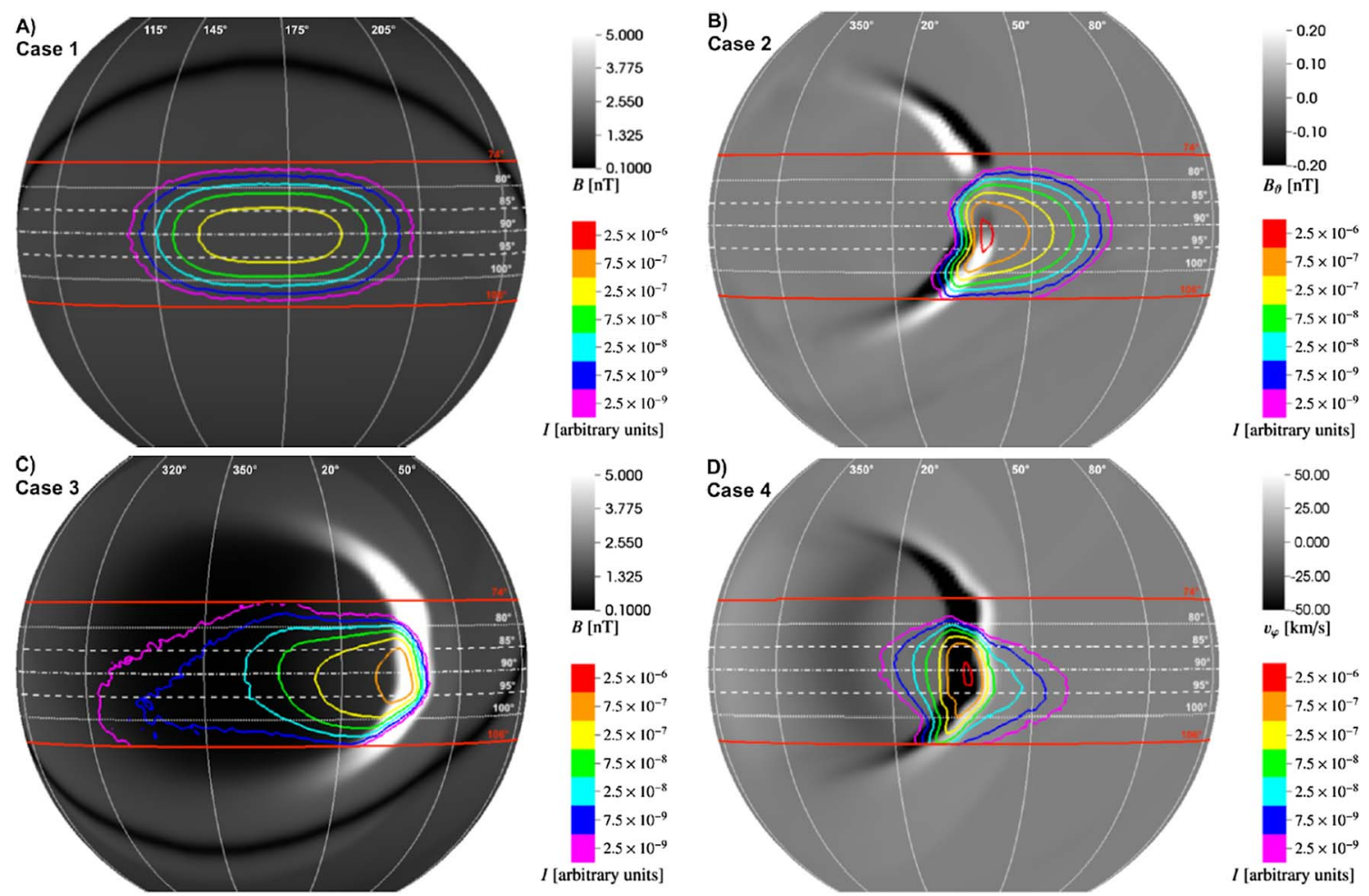

Fig. 3. Contour plots of the particle intensity at $r=1.5 \mathrm{AU}$, drawn on top of different MHD solar wind variables, $15.5 \mathrm{~h}$ after particle injection and for the simulations with cross-field diffusion. The red parallels indicate the borders of the sampling region. Four cases with different injection regions are shown. Upper left: intensities of case 1 drawn on top of the magnetic field magnitude. Upper right panel: intensities of case 2 drawn on top of the magnetic field colatitude component. Lower left panel: intensities of case 3 drawn on top of the magnetic field magnitude. Lower right panel: intensities of case 4 drawn on top of the longitudinal velocity component. (Wijsen et al., 2019b).

3-D particle focused transport model coupled with the EUHFORIA solar wind model to describe impulsive SEP events in non-nominal solar wind conditions in the interplanetary domain (Wijsen et al., 2019a, b). Test-particle approaches are also the way to make the Monte Carlo modelling compatible with operational requirements. From this variety of models, we will select the best compromise between accuracy and efficiency.

Objective 4: Developing an operational prediction tool for GICs in the EU power grid.

To provide a realistic description of the ionospheric medium and to determine ionospheric horizontal electrical currents, the CNRS group will use the electrodynamics model IMM (Hurtaud et al., 2007), which will be coupled to the firstprinciples ionosphere model IPIM at both high- and midlatitudes (Blelly et al., 1996, 2005; Marchaudon \& Blelly, 2015). All these models have been developed in the IRAP/ CNRS group and have been successfully coupled in the past (Blelly, 2003). Fed with sufficiently accurate energy inputs, e.g. from the solar wind, the coupled models give an excellent description of ionospheric dynamics at speeds suitable for operational space weather forecasting and will provide accurate ionospheric conductivities and currents.

We will also develop and couple a Biot-Savart model to these different models to provide forecasts of geomagnetic variations at any point on the ground. Using this forecast model, the BGS team will simulate the flow of GICs, induced by rapid, high-amplitude magnetic field changes, in national models that are part of the connected European and separate UK electrical transmission systems and determine the impact on electrical substations within these networks, including impacts within individual transformers at key locations. We will build on previous work (e.g. Thomson et al., 2005; Kelly et al., 2017; EU FP7 "EURISGIC") through updated Earth conductivity models for Europe and the UK and updated electrical network details that allow us to probe transformer level impacts at key substation sites accurately.

To provide context and comparison the BGS group will compare the results of the coupled EUHFORIA/CNRS model, in terms of prediction accuracy of $\mathrm{d} B / \mathrm{d} t$ and predicted GIC, with the $\mathrm{d} B$ output of an existing and tested geospace model, OpenGGCM (Raeder et al., 2017), and, independently, a statistical model of 30-min predicted peak $\mathrm{d} B / \mathrm{d} t$ (Wintoft et al., 2015). These $\mathrm{d} B$ and $\mathrm{d} B / \mathrm{d} t$ predictions will be coupled to a detailed UK power grid network model, as a representative model for a complex national system within Europe.

Objective 5: Developing more reliable prediction tools for harsh radiation in geospace.

In order to provide a realistic description of the radiation dose in silicon and tissue-equivalent material aboard the ISS and at aircraft altitudes, a concept that has successfully applied to neutron monitor (NM) measurements (Bieber et al., 2004; Heber et al., 2015) and dose rate computations (Mishev \& Usoskin, 2015) will be adapted. The approach used to interpret 
the NM data is based on so-called yield functions (CaballeroLopez, 2016) which are computed by tracking particles through the atmosphere and determine the NM response to the radiation environment caused by these particles. Different programs based on the GEANT4 (Agostinelli et al., 2003) or CORSIKA library (Heck et al., 1998) have been utilised computing the yield function (see Caballero-Lopez, 2016). However, the yield function in the rigidity range between 1 and $16 \mathrm{GV}$ can be determined experimentally by latitudinal surveys (CaballeroLopez \& Moraal, 2012). We will follow a mixed approach. In order to determine the yield function for the radiation dose in silicon, we will analyse DOSTEL measurements aboard the ISS (see Labrenz et al., 2015) and aboard an aircraft (Möller et al., 2012) using galactic cosmic ray spectra inferred from O'Neill (2010). In order to determine the yield function in tissue-equivalent material we will set up a GEANT4 model of the DOSTEL within the radiation environment that reproduces the yield in the range from $1 \mathrm{GV}$ to $16 \mathrm{GV}$. Using our (coronal + interplanetary) SEP transport model together with the detailed computation of motion of charged particles in the variable Earth's magnetic field (Desorgher, 2005), we will compute the radiation dose in silicon and in tissue during a SEP event within the ISS and on typical polar routes.

Objective 6: Creating completely novel space weather forecasting service facilities.

To maximise the impact of the project, we want to distribute the science, software and services developed within the project to target groups that have an interest or are impacted by space weather in general. Therefore, we will disseminate a message tailored to the needs of a stakeholder or client making use of the appropriate tactics and tools. We want to raise awareness, reach involvement and come to a possible future collaboration.

Presently, various CME catalogues exist, but most of them focus only on one type of observation/instrumentation, typically based on white-light coronagraph imaging. These catalogues also typically provide rather basic CME parameters that are subject to projection effects. A significant step in the direction of presenting combined and community-wide catalogues was established in the FP7-funded HELCATS project (https:// www.helcats-fp7.eu). We will use realistic information of CME coronal parameters (Objective 2) to constrain flux ropes in EUHFORIA, provided by different advanced reconstruction techniques and data-driven modelling that apply a wide variety of state-of-the-art remote-sensing observations and also upcoming data. The results will be compared to the real data, in terms of metrics for continuous and binary variables. Initial preliminary comparisons have been done by Scolini et al. (2019).

For shocks, we will apply EUV and radio triangulation to reconstruct the shock geometry. The radio triangulation techniques use direction-finding observations of the SWAVES instruments on-board WIND and STEREO spacecraft. As WIND is a spinning spacecraft and STEREO is a 3 -axis stabilised spacecraft, different direction-finding methods will be used for these spacecraft (Magdalenic et al., 2014). The results of radio triangulation will be combined with white-light based reconstruction techniques in order to provide the 3D picture of the CME and the radio-emitting part of the CME-driven shock wave. We will make use of radio-tracking of CMEs using Type II bursts. Using the Vršnak et al. (2004) density model, we will compile the distance maps of the CME-driven shock waves.

\section{Implementation and first results}

\subsection{Some first results of the project}

The EUHFORIA outreach website is online: https:// euhforia.com/ and contains information on the EUHFORIA 2.0 project and on the EUHFORIA model itself, and links to the Blog and the Wiki. It also contains a link to the EUHFORIA Online app (https://www.euhforiaonline.com/). It provides a graphical user interface (GUI) to set all the input parameters to run EUHFORIA Corona and EUHFORIA Heliosphere and provides the standard output pictures and movies automatically.

Below we briefly present some of the first scientific results obtained. Papers with more detailed descriptions and discussions of these results, have been submitted or are in preparation.

\subsubsection{Global non-potential model of the coronal magnetic field}

The development of a global model of the coronal magnetic field as an alternative to the current PFSS + Schatten current sheet model in EUHFORIA, has started. The new model is based on the magneto-frictional method (MFM) for timedependent data-driven modelling of active region evolution that has been developed by Pomoell et al. (2019). For the global coronal magnetic field, the MFM code has been extended to support spherical geometry. Preliminary tests with the new code have been performed. Relaxation of an initial dipolar magnetic field to include the effect of stretching of field lines due to the solar wind has been successfully performed. The resulting magnetic field structure resembles closely those obtained from MHD-based coronal models, incl. an open streamer belt (Fig. 4).

As an example application of more advanced boundary conditions, a second test involving the energization of the coronal magnetic field via build-up of currents in the coronal magnetic field has also been carried out. An example is illustrated in Figure 5, showing a snapshot of the coronal magnetic field with the formation of a sheared arcade structure in a multipolar magnetic field structure (for the full animation, see Supplementary Material, Streamer_shear_3d_view). The MFM approach allows to perform such computations very rapidly (a couple of minutes on a laptop for axisymmetric cases) in contrast to much more costly MHD-based methods. This allows timedependent modeling of the coronal magnetic field to be performed at a reasonable computational cost. Such modeling is also radically different from PFSS as the latter does not include currents in the model solution. Currently, methods of specifying the low-coronal boundary conditions driving the evolution based of the methods of Lumme et al. (2017) are being evaluated.

\subsubsection{Multi-VP model coupled to EUHFORIA}

The physics-based model Multi-VP (Pinto \& Rouillard, 2017) first makes a PFSS extrapolation of a magnetogram and then solves the system of MHD equations describing the heating and acceleration of a wind stream along a given magnetic flux-tube. Every such flux tube is thus a 1D MHD wind solution. This is illustrated in Figure 6 using the WSO magnetogram for CR2056 (2007 April-May) and showing a sample of 

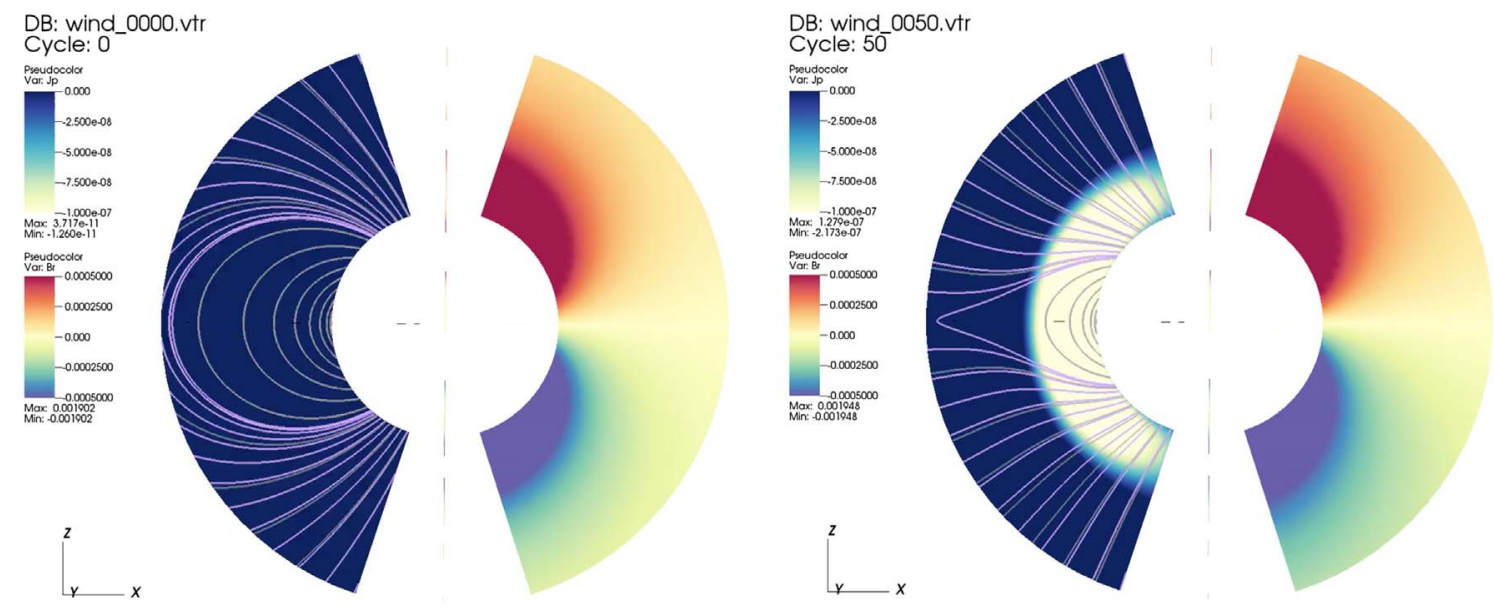

Fig. 4. Magneto-frictional relaxation simulation of a dipolar magnetic field to include the stretching of the field due to solar wind outflows. At the left, the initial state of the simulation is shown, while on the right, the relaxed state at the end of the simulation is shown.

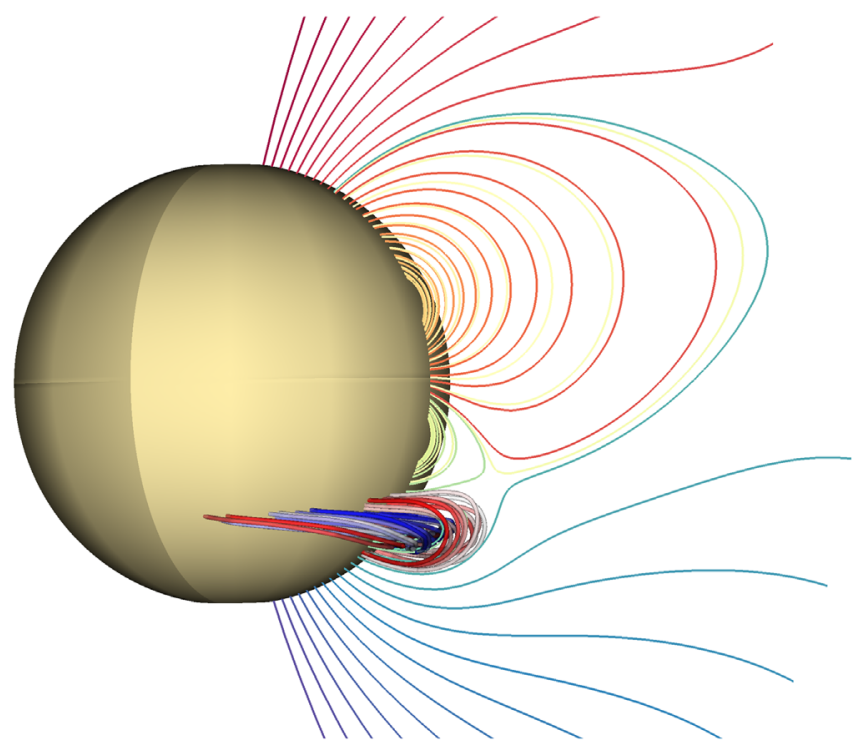

Fig. 5. Formation of a sheared arcade structure in a multipolar magnetic field structure. The evolution of the Coronal field is efficiently computed using the MFM developed at University of Helsinki. This figure is a frame from a movie that is published as Supplementary Material to this paper.

magnetic field lines obtained via PFSS extrapolation, which are used to initiate the model. Eventually, the total of all these 1D solutions samples the whole solar atmosphere. Interpolation of the results on a grid on a sphere at $0.1 \mathrm{AU}$, produces the MHD input file with density, pressure, magnetic field and radial velocity distribution required for the heliospheric part of EUHFORIA.

In the framework of the ongoing validation of the solar wind modelling with EUHFORIA, we implemented and tested the MULTI-VP model as an alternative coronal model, i.e. as an alternative boundary condition for the heliospheric wind simulation in EUHFORIA. In other words, we replaced the semiempirical WSA + SCS based coronal model in EUHFORIA by Multi-VP, and coupled it to the heliospheric wind model

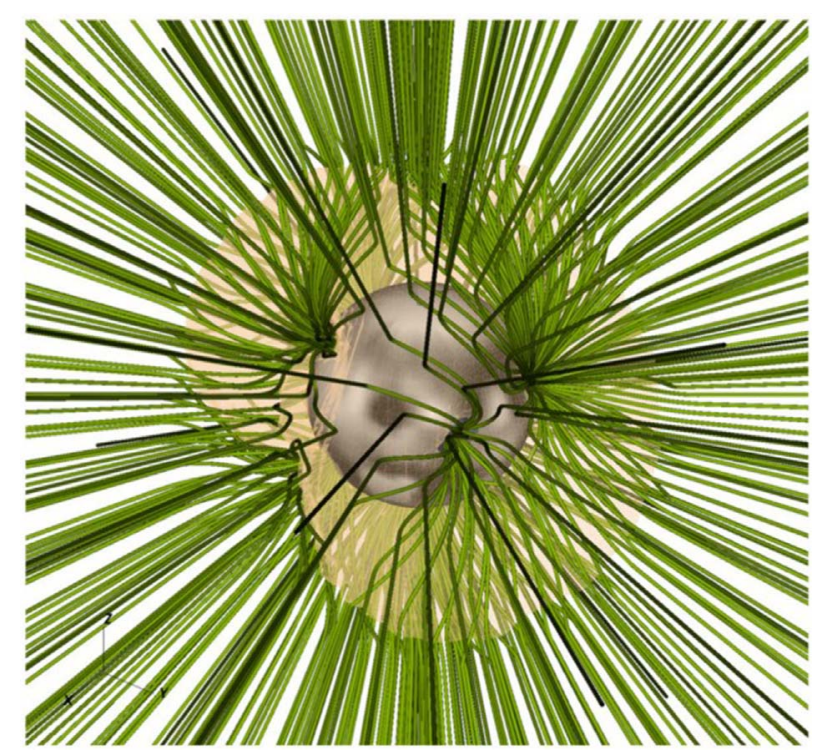

Fig. 6. The grey scale on the solar surface indicates the input WSO magnetogram in the MULTI-VP model for CR2056 (2007 AprilMay). A sample of magnetic field lines obtained via PFSS extrapolation used to initiate the model, are also depicted. The transparent yellow surface indicates the coronal hole boundaries (closed-field regions are excluded from the domain). For more details see Pinto \& Rouillard (2017).

in EUHFORIA. In doing so, some difficulties appear as there are a number of sub-Alfvénic speeds at $0.1 \mathrm{AU}$ in the MultiVP output. These need to be transformed to (super-)Alfvénic because the boundary conditions programmed in the heliospheric model assume that all boundary velocities are superAlfvénic. Therefore, the sub-Alfvénic pixels were replaced by interpolations using their first super-Alfvénic neighbors while obeying the mass-flux conservation.

The first results and comparisons of EUHFORIA modelled output at Earth produced by employing the WSA + SCS and MULTI-VP coronal models have been obtained. The MultiVP based boundary conditions turn out to better capture the fast 


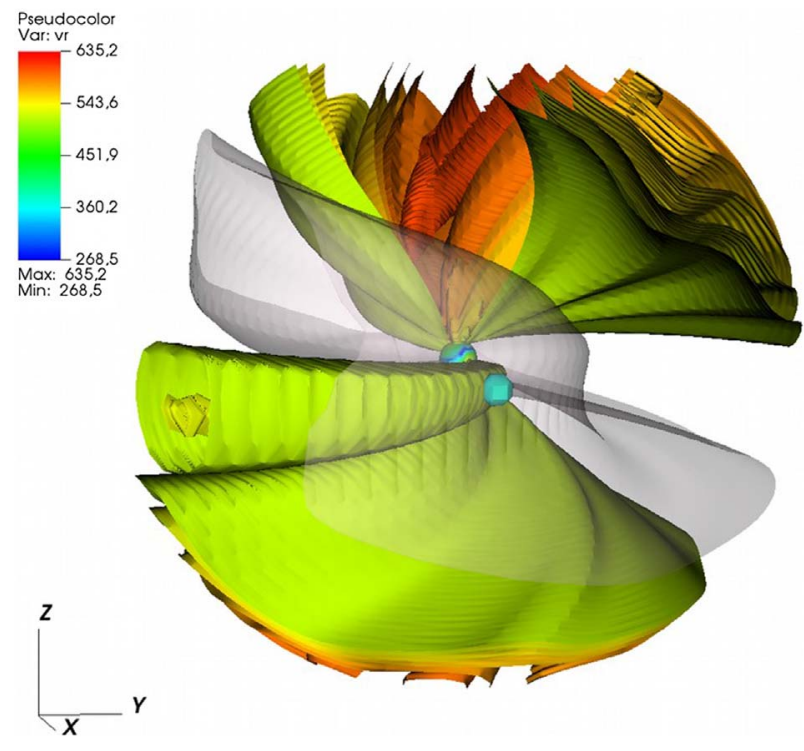

Fig. 7. 3D visualization of the structures produced by MULTIVP + EUHFORIA-heliosphere throughout the inner heliospheric domain for the solar minimum test case. The heliospheric current sheet is indicated in grey while the colorful isosurfaces represent solar wind speeds between 520 and $600 \mathrm{~km} / \mathrm{s}$. A demonstration of the spherical inner boundary surface can be seen in the middle of the domain. It depicts the radial velocities at 0.1 AU. Earth is shown in light blue color.

solar wind streams. Figure 7 shows a 3D visualization of the structures produced by MULTI-VP + EUHFORIA-heliosphere throughout the inner heliospheric domain for a solar minimum test case. The heliospheric current sheet is indicated in grey while the colorful isosurfaces represent solar wind speeds between 520 and $600 \mathrm{~km} / \mathrm{s}$. A demonstration of the spherical inner boundary surface can be seen in the middle of the domain. It depicts the radial velocities at 0.1 AU. The Earth is shown in light blue color and it can be seen that a fast solar wind stream hits the Earth, which is also seen in WIND data. The standard EUHFORIA set-up with the WSA + SCS coronal model, however, does not capture this fast wind stream at Earth, regardless of the magnetogram used. Another HSS case during maximum, showed similar results. Samara et al. (2020) showed that the choice of the coronal model as well as the choice of the magnetogram play an important role on the quality of the solar wind forecast and conclude that a statistical analysis is needed to confirm these findings.

\subsubsection{Alternative CME flux-rope models}

The current spheromak CME model in EUHFORIA (Scolini et al., 2019, 2020; Verbeke et al., 2019) significantly improves the predictions at L1 as compared to the "standard" cone CME model. Especially the magnetic field component predictions are much better and this, in turn, yields better predictions of the geo-effectiveness of the CME impacts (Scolini et al., 2020). However, the latter turns out to be true only in case of a "full hit", when the "nose" of the CME hits the Earth. When the Earth is hit by a flank or "leg" of the CME, the event cannot be modelled very well with a spheromak model as this



Fig. 8. Snapshot of EUHFORIA simulation employing a toroidal flux rope currently being tested at University of Helsinki. The CME propagates at the interface of a slow and fast solar wind. The field line connected to Earth is shown as the thick blue/green curve and exhibits a complex connectivity with the flux rope magnetic field. The flux rope experiences significant asymmetric erosion, being more prominent at the western flank in this case.

model does not have the typical flux-rope shape of the CMEs. Therefore, we first implemented the Fri3D model (Isavnin, 2016) as an alternative flux-rope CME model and this model is currently being tested (verification of the modelling results and robustness of the implementation) before it will be committed to the main EUHFORIA branch. A paper on the integration of the Fri3D flux-rope CME model in EUHFORIA is in preparation.

An alternative toroidal flux-rope CME model has been implemented already and is also currently being tested. The preliminary results show improved connectivity and magnetic field profiles compared to the current spheromak-based model. As a matter of fact, this model also has the typical flux-rope shape of the CMEs and enables one to keep the CMEs connected to the Sun, as illustrated in Figure 8 which shows a snapshot of an EUHFORIA simulation with this novel CME model. In this particular case, the CME propagates at the interface of two slow and fast solar wind sections. The field line connected to the Earth is shown as the thick blue/green curve (with the small sphere indicating the position of the Earth). It can be seen that is exhibits a complex connectivity with the flux-rope magnetic field. During its evolution through the heliosphere, the flux-rope experiences significant asymmetric erosion, being more prominent at the western flank in this case, due to the interaction with the fast solar wind section.

\subsubsection{Including solution adaptive mesh refinement techniques in EUHFORIA}

We also started working on a finite volume method based implementation based on MPI-AMRVAC using a grid co-rotating with the Sun so that the obtained steady background 

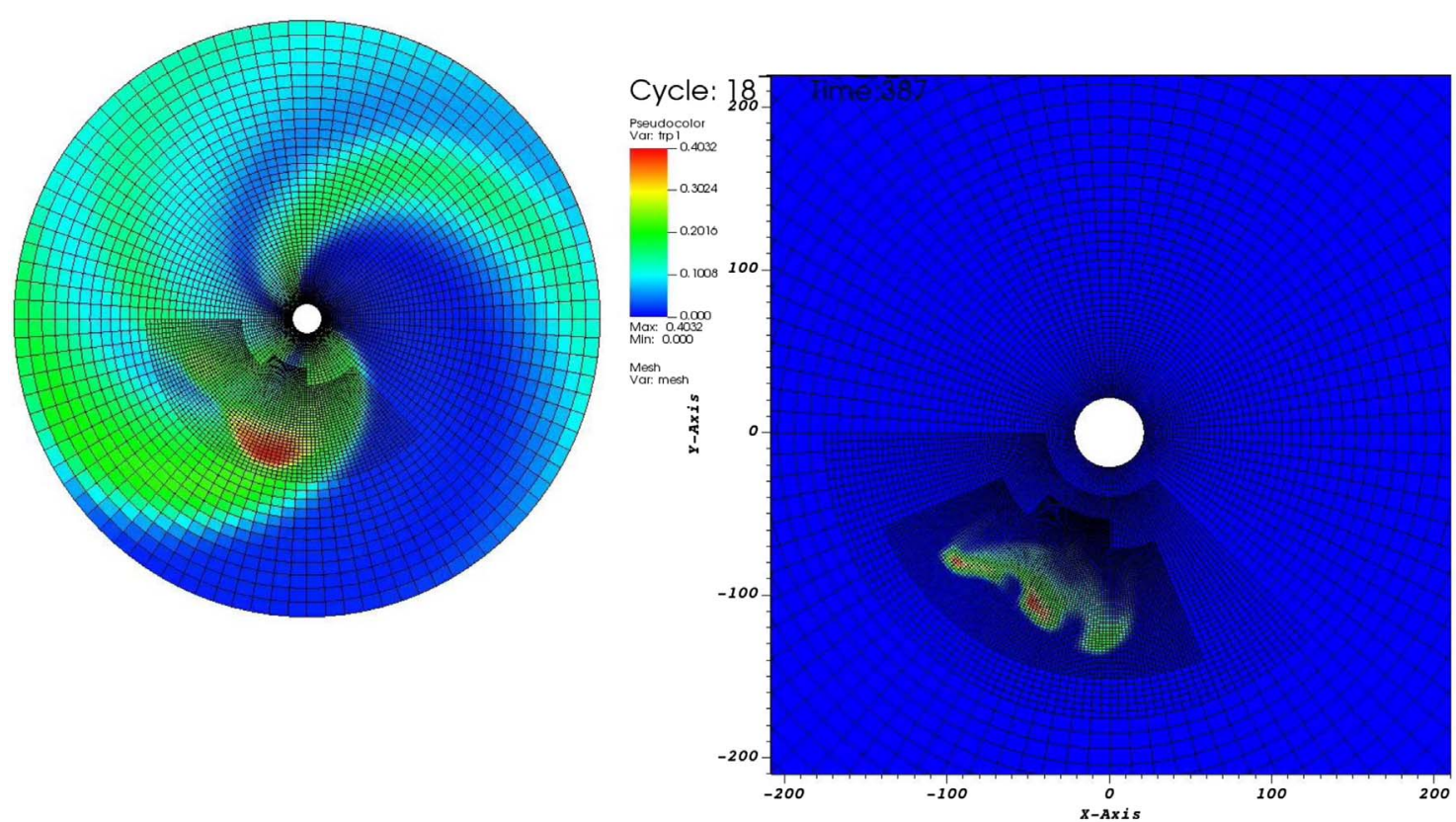

Fig. 9. Left: snapshot of a two-level solution adapted mesh for a cone CME in a stretched grid using TVDLF. Right: another example snapshot of a different test with three levels of AMR.

wind is time-independent, unlike the current EUHFORIA set up using HEEQ coordinates, i.e. in which the Sun rotates in the grid and the Earth has a fixed longitude. A stretched grid has been implemented for the background wind and the effect of grid stretching combined with solution adaptive mesh refinement (AMR) on the steady solar wind and evolving CMEs are being investigated. Grid stretching is especially useful in spherical geometries, because when the values of $\Delta r, \Delta \theta$, and $\Delta \varphi$ (using spherical coordinates $(r, \theta, \varphi)$ ) are constant, the cell widths become ever larger the further away from the grid center while the radial cell length stays the same. This results in deformed grid cells which affects the numerical accuracy. Applying grid stretching results in more regular "cubic" grid cells resulting in a better accuracy. Moreover, the simulation is faster on a stretched grid because there are much fewer cells needed in the radial direction.

The first results have been verified (comparison with nonstretched grid results, timings, convergence study, adjusted visualization, etc.) on realistic winds (based on magnetogram extrapolations). Also, the cone CME model has already been implemented and is currently being tested and convergence studies have been done.

Next, different AMR strategies are tested and timed, i.e. with AMR thresholds on different quantities like density gradient, tracing function (tracing the CME plasma), velocity divergence (which is negative at CIR and CME shocks, i.e. where particles can get accelerated and AMR is thus useful), etc. and combinations of these, in order to fine-tune the AMR both on the CIR shocks in the background solar wind and at the CME shock wave and magnetic cloud (to study erosion and deformation, for instance). The results are very encouraging: the stretching of the grid yields a better performance and speed-ups of 2.23-2.8 were obtained, depending on the resolution. Combining AMR with grid stretching is much more efficient. The performed tests yielded a speed-up of 13.97 using

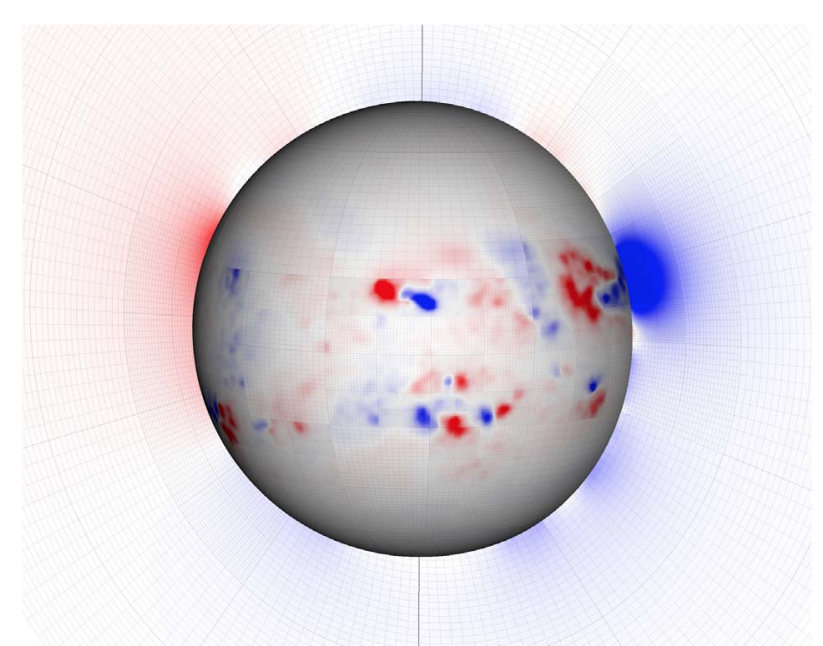

Fig. 10. Preliminary example of the coronal model computed on an AMR grid using constrained transport - supported software being developed at University of Helsinki.

two grid levels (i.e. one refinement level) and up to 99 when using three grid levels, limiting the higher resolution to the regions where necessary. However, these speed-ups of course depend on the case under study and on the refinement criteria applied. For instance, when there are multiple CIRs and/or multiple CMEs, much more refinement area will be required and the speed-up is lower.

Figure 9 (left) shows a snapshot of such a CME evolution case using a two-level solution adapted mesh for a cone CME in a stretched grid using a TVDLF solver. On the right-hand side in this figure, another example is shown with a snapshot of a different test using three levels of AMR in a more complex 

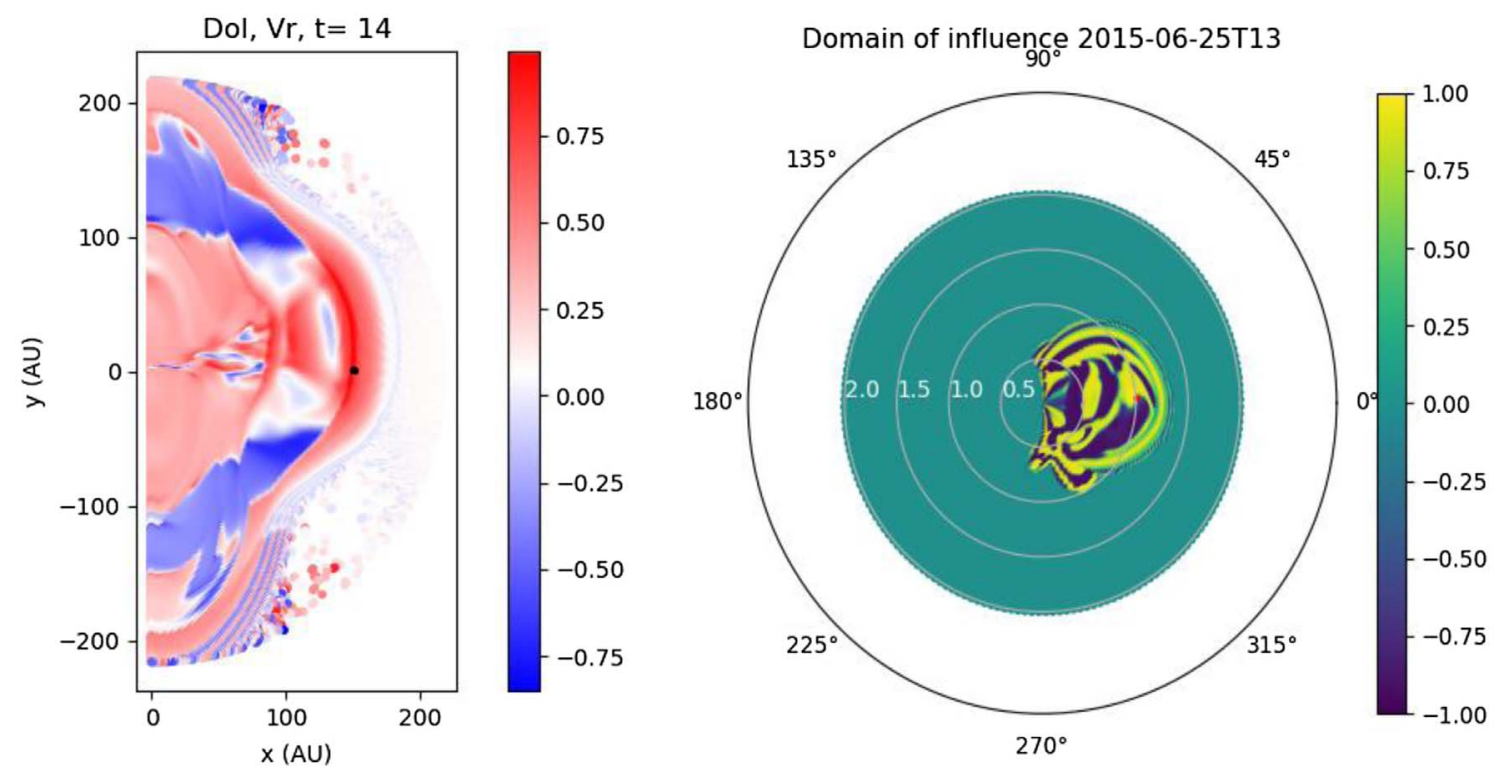

Fig. 11. Left: domain of Influence calculated from a PLUTO ensemble, using the radial velocity as a criterion, in the meridional plane. The perturbed quantities are the radial velocity of the CME and its size. Right: same, from an EUHFORIA ensemble, image on the meridional plane.

case where the CME is launched on an interaction of a slow and a fast wind region. The colors correspond to the radial velocity component

The University of Helsinki team is also working on an alternative AMR strategy in the current Constrained Transport scheme which guarantees the solenoidal condition (Div $(\boldsymbol{B})=0)$ to be satisfied up to machine accuracy. A preliminary example of the coronal model computed on an AMR grid is shown in Figure 10, for a complicated case with many active regions.

\subsubsection{Evaluation of data-assimilation based on Kalman filtering for wind modelling}

We applied a Representer and Domain of Influence analysis (Bennett, 1992; Echevin et al., 2000; Evensen, 2009; Skandrani et al., 2014), which are powerful statistical tools that enable to estimate the effectiveness of data assimilation techniques when applied to a specific code or model, even before assimilating actual data. Representer analyses based on the "Domain of Influence" (DoI) have already been tested on several different problems related to space weather. The cases examined are the propagation of a CME against a background solar wind using the codes EUHFORIA (in full 3D), and the propagation of a CME against a background solar wind using the PLUTO code (in axisymmetric 2.5D simulations), illustrated in Figure 11. The left panel of this figure shows the domain of influence calculated from a PLUTO ensemble, using the radial velocity as a criterion, in the meridional plane. The perturbed quantities are the radial velocity of the CME and its size. This step was taken as a preliminary build-up phase to develop the tools in a reduced dimensionality case. The right panel in Figure 11 shows the domain of influence calculated from an EUHFORIA ensemble, but this time in the equatorial plane.

All tests use an ensemble of simulations, at least 50, where each member of the ensemble is modified (compared to the reference run) using a perturbation selected from a Gaussian. We then calculate the variance and the correlation of the ensemble using a physical quantity (e.g. velocity) as a criterion.

In the EUHFORIA ensembles specifically, we first model the background solar wind using real magnetograms. Then we inject a cone CME with different velocity and size in each simulation. We tested additionally low- and high-resolution runs. We are currently examining the effect of perturbations in the magnetograms.

The results of this study have been submitted for publication on a special issue of Frontiers in Astrophysics dedicated to space weather modelling. The related paper is under review (Millas et al., 2020).

\subsubsection{Integration of SEP models - feasibility study}

The SEP modelling approaches being developed by the team members have been evaluated with respect to their potential to be applied in EUHFORIA 2.0. Regarding the transport modelling of SEPs, there are three simulation models available, as mentioned before: the PARADISE code of the KU Leuven, the DSPM code of the University of Turku, and the $\mathrm{SaP}$ code of the University of Barcelona. The SaP code requires the least CPU time and does seem to be the most appropriate to be integrated to obtain an operational model. However, it has been assessed that among these transport models, PARADISE has the broadest range of applicability in the various complex conditions that can take place in interplanetary space. Therefore, the next step has been to explore whether PARADISE, which uses forward Monte Carlo integration in time, is fast enough and can be applied directly or needs to be made more efficient.

We have performed a scaling-test of the PARADISE model, using Skylake and the Broadwell processor architectures available on Tier-1 of the Flemish supercomputer (VSC). The results are depicted in Figure 12. For these simulations, protons were 


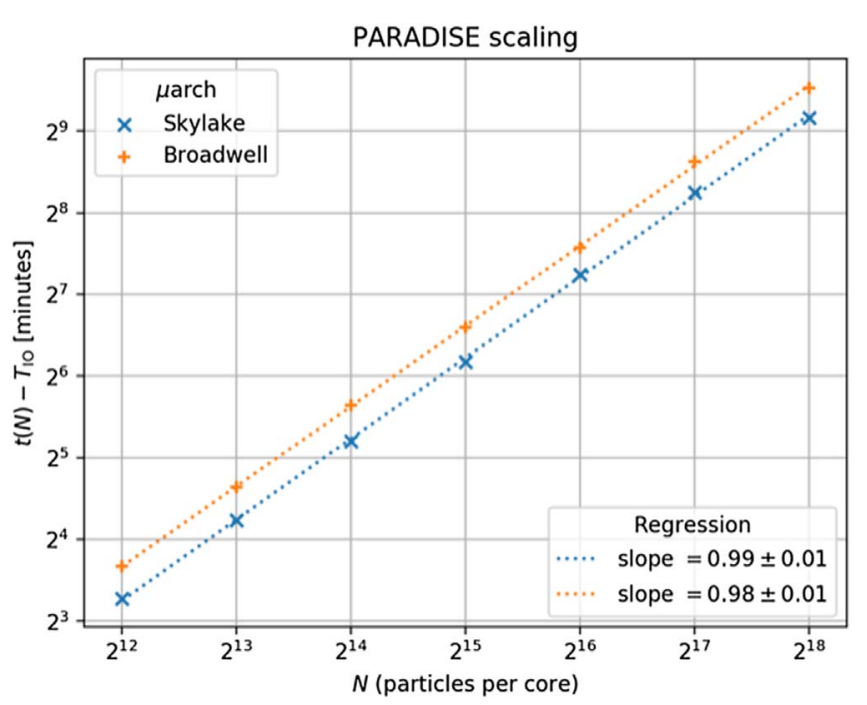

Fig. 12. PARADISE scaling test results.

integrated forward in time for a physical time of $42 \mathrm{~h}$, using high-resolution runs of EUHFORIA as input. The solar wind was updated in PARADISE with a cadence of 5 min (physical time) on a grid with $1024 \times 80 \times 360$ grid points, leading to an $\mathrm{I} / \mathrm{O}$ bound of $T_{\mathrm{IO}} \sim 47 \mathrm{~min}$ and $T_{\mathrm{IO}} \sim 62 \mathrm{~min}$ on the Skylake and Broadwell architectures, respectively. The figure below illustrates that PARADISE is an entirely parallel program, which is to be expected as there is no interaction between the simulated test-particles, and hence there is no communication necessary between the cores. Different options to reduce the I/O bound are being investigated, including the use of stretched grids in the radial direction, reducing the latitudinal extent of the grid, and increasing the snapshot cadence.

\section{Brief preliminary conclusion}

The EU H2020-SPACE-2019 project EUHFORIA 2.0 started in December 2019 and involves eleven research teams, supported by an "International Expert Advisory Panel". The project will develop an advanced space weather forecasting tool, combining an MHD solar corona and wind model with one or more SEP models. The tool will be applied to study the geoeffectiveness of the impacts of CMEs, CIRs and SEPs and will help to mitigate (part of) the damage these cause. Extreme events will also be considered, though the emphasis will be on improving the prediction of daily space weather and its effects. In particular, the effects on forecasting Geomagnetically Induced Currents and radiation on geospace will be addressed. The first results, obtained within the first six months of the project, have been presented and the project is on schedule. The final innovative tool will be integrated into both the Virtual Space Weather Modelling Centre (ESA) and the space weather forecasting procedures at the ESA SSCC in Ukkel (Belgium), so that it will be available to the space weather community and effectively used for improved predictions and forecasts of the evolution of CME magnetic structures and their impact on Earth.

\section{Supplementary Material}

Supplementary material is available at http://www.swscjournal.org/10.1051/swsc/2020055/olm

Movie 1. Streamer_shear_3d_view.

Acknowledgements. This project has received funding from the European Union's Horizon 2020 research and innovation programme under grant agreement No. 870405. Additional support from the projects $\mathrm{C} 14 / 19 / 089$ (C1 project Internal Funds KU Leuven), G.0D07.19N (FWO-Vlaanderen), C 90347 (ESA Prodex), Belspo BRAIN project BR/165/A2/ CCSOM is greatly acknowledged. For the computations at KU Leuven we used the infrastructure of the VSC - Flemish Supercomputer Center, funded by the Hercules foundation and the Flemish Government - department EWI.

\section{References}

Afanasiev A, Vainio R. 2013. Monte Carlo simulation model of energetic proton transport through self-generated Alfvén waves. Astrophys J Suppl Ser 207: 29. https://doi.org/10.1088/0067-0049/ 207/2/29.

Afanasiev A, Vainio R, Kocharov L. 2014. The effect of stochastic re-acceleration on the energy spectrum of shock-accelerated protons. Astrophys J 790: 36. https://doi.org/10.1088/0004-637X/ 790/1/36.

Afanasiev A, Battarbee M, Vainio R. 2015. Self-consistent Monte Carlo simulations of proton acceleration in coronal shocks: Effect of anisotropic pitch-angle scattering of particles. A\&A 584: 81 . https://doi.org/10.1051/0004-6361/201526750.

Afanasiev A, Aran A, Vainio R, Rouillard A, Zucca P, Lario D, Barcewicz S, Siipola R, Pomoell J, Sanahuja B, Malandraki OE. 2018a. Modelling of shock-accelerated gamma-ray events. Astrophys Space Sci Libr 444: 157. https://doi.org/10.1007/978-3-31960051-2_9.

Afanasiev A, Vainio R, Rouillard AP, Battarbee M, Aran A, Zucca P. $2018 \mathrm{~b}$. Modelling of proton acceleration in application to a ground level enhancement. $A \& A$ 614: 4. https://doi.org/10.1051/00046361/201731343.

Agostinelli S, Allison J, Amako K, Apostolakis J, Araujo H, et al. 2003. GEANT4 - a simulation toolkit. Nucl Instrum Methods Phys Res A 506: 250-303. https://doi.org/10.1016/S0168-9002(03) 01368-8.

Bacchini F, Olshevsky V, Poedts S, Lapenta G. 2017. A new particlein-cell method for modeling magnetized fluids. Comput Phys Comm 210: 79-91. https://doi.org/10.1016/j.cpc.2016.10.001.

Bennett AF. 1992. Inverse methods in physical oceanography, Cambridge University Press, Cambridge, England. ISBN-13: 9780521055284.

Bieber JW, Evenson P, Dröge W, Pyle R, Ruffolo D, Rujiwarodom M, Tooprakai P, Khumlumlert T. 2004. Spaceship earth observations of the easter 2001 solar particle event. Astrophys J Lett 601: L103-L106. https://doi.org/10.1086/381801.

Blelly P-L. 2003. SpaceGRID study final report. SGD-SYS-DATTN-100-1.2. Issue 1.2. https://www.yumpu.com/en/document/ $\mathrm{read} / 50039475 /$ final-report-rss-esa.

Blelly P-L, Robineau A, Lilensten J, Lummerzheim D. 1996. 8-moment fluid models of the terrestrial high latitude ionosphere betweeen 100 and 3000 km. In: Solar terrestrial energy program 
ionospheric model handbook, Schunk RW (Ed.), Utah State Univ., Logan, pp. 53-72. https://www.bc.edu/content/dam/bc1/offices/ ISR/SCOSTEP/Multimedia/other/ionospheric-models.pdf.

Blelly P-L, Lathuillère C, Emery B, Lilensten J, Fontanari J, Alcaydé D. 2005. An extended TRANSCAR model including ionospheric convection: Simulation of EISCAT observations using inputs from AMIE. Ann Geophys 23: 419-431. https://doi.org/10.5194/angeo23-419-2005.

Caballero-Lopez RA. 2016. An estimation of the yield and response functions for the mini neutron monitor. JGR (Space Phys) 121: 7461-7469. https://doi.org/10.1002/2016JA022690.

Caballero-Lopez RA, Moraal H. 2012. Cosmic-ray yield and response functions in the atmosphere. JGR: Space Phys 117: A12103. https://doi.org/10.1029/2012JA017794.

Desai M, Giacalone J. 2016. Large gradual solar energetic particle events. Living Rev Sol Phys 13: 3. https://doi.org/10.1007/s41116016-0002-5.

Desorgher L. 2005. PLANETOCOSMICS software user manual issue 0.1, 2006-06-14, http://cosray.unibe.ch/ laurent/planetocosmics/ doc/planetocosmics_sum.pdf.

Echevin V, De Mey P, Evensen G. 2000. Horizontal and vertical structure of the representer functions for sea surface measurements in a coastal circulation model. J Phys Oceanogr 30: 2627-2635. https://doi.org/10.1175/1520-0485(2000)030<2627:HAVSOT> 2.0.CO;2.

Evensen G. 2009. Data assimilation: The ensemble Kalman filter, Springer Science \& Business Media, Berlin, Germany. ISBN 9783-642-03711-5.

Farrugia CJ, Jordanova VK, Thomsen MF, Lu G, Cowley SWH, Ogilvie KW. 2006. A two-ejecta event associated with a two-step geomagnetic storm. JGR: Space Phys 111: A11. https://doi.org/ 10.1029/2006JA011893.

Fisk LA, Lee MA. 1980. Shock acceleration of energetic particles in corotating interaction regions in the solar wind. Astrophys $J$ 237: 620-626. https://doi.org/10.1086/157907.

Heber B, Galsdorf D, Herbst K, Gieseler J, Labrenz J, et al. 2015. Mini neutron monitor measurements at the Neumayer III station and on the German research vessel Polarstern. J Phys: Conf Ser 632: 012057. https://doi.org/10.1088/1742-6596/632/1/012057.

Heck D, Knapp J, Capdevielle J, Schatz G, Thouw T. 1998. CORSIKA: A Monte Carlo code to simulate extensive air showers, Forschungszentrum Karlsruhe $\mathrm{GmbH}$, Karlsruhe (Germany), V + 90 p. TIB Hannover, D-30167 Hannover. Bibcode: 1998cmcc. book......H.

Hurtaud Y, Peymirat C, Richmond AD. 2007. Modeling seasonal and diurnal effects on ionospheric conductances, region-2 currents, and plasma convection in the inner magnetosphere. JGR 112: A09217. https://doi.org/10.1029/2007JA012257.

Isavnin A. 2016. FRiED: A novel three-dimensional model of coronal mass ejections. Astrophys J 833: 267. https://doi.org/ 10.3847/1538-4357/833/2/267.

Kelly GS, Viljanen A, Beggan CD, Thomson AWP. 2017. Understanding GIC in the UK and French high-voltage transmission systems during severe magnetic storms. Space Weather 15: 99114. https://doi.org/10.1002/2016SW001469.

Kilpua EKJ, Olspert N, Grigorievskiy A, Käpylä MJ, Tanskanen EI, et al.. 2015. Statistical study of strong and extreme geomagnetic disturbances and solar cycle characteristics. Astrophys J 806: 272. https://doi.org/10.1088/0004-637X/806/2/272.

Labrenz J, Burmeister S, Berger T, Heber B, Reitz G. 2015. Matroshka DOSTEL measurements onboard the International
Space Station (ISS). J Space Weather Space Clim. 5: A38. https://doi.org/10.1051/swsc/2015039.

Lario D, Simnett GM. 2004. Solar energetic particle variations. In: Solar variability and its effects on climate, geophysical monograph, Vol. 141, pp. 195-216. https://doi.org/10.1029/141GM14.

Liu YD, Hu H, Wand R, Yand Z, Zhu B, Liu YA, Luhman JG, Richardson JD. 2015. Plasma and magnetic field characteristics of solar coronal mass ejections in relation to geomagnetic storm intensity and variability. Astrophys J Lett 809: L34. https://doi.org/ 10.1088/2041-8205/809/2/L34.

Liu J, Ye Y, Shen C, Wang Y, Erdélyi R. 2018. A new tool for CME arrival time prediction using machine learning algorithms: CATPUMA. Astrophys J 855: 109. https://doi.org/10.3847/1538-4357/ aaae69.

Lumme E, Pomoell J, Kilpua EKJ. 2017. Optimization of photospheric electric field estimates for accurate retrieval of total magnetic energy injection. Sol Phys 292: A191. https://doi.org/ 10.1007/s11207-017-1214-0.

Magdalenić J, Marqué C, Krupar V, Mierla M, Zhukov AN, Rodriguez L, Maksimović M, Cecconi B. 2014. Tracking the CME-driven shock wave on 2012 March 5 and radio triangulation of associated radio emission. Astrophys J 791: 115. https://doi.org/ 10.1088/0004-637X/791/2/115.

Marchaudon A, Blelly P-L. 2015. A new interhemispheric 16-moment model of the plasmasphere-ionosphere system: IPIM. JGR: Space Phys 120: 5728-5745. https://doi.org/10.1002/2015JA021193.

Millas D, Innocenti M.E, Laperre B, Raeder J, Poedts S, Lapenta G. 2020. The effectiveness of data assimilation in space weather forecasting: Heliospheric and magnetospheric applications via MHD simulations. Front Astron Space Sci Stellar Solar Phys 7: 571286. https://doi.org/10.3389/fspas.2020.571286.

Mishev A, Usoskin I. 2015. Numerical model for computation of effective and ambient dose equivalent at flight altitudes. Application for dose assessment during GLEs. J Space Weather Space Clim 5: A10. https://doi.org/10.1051/swsc/2015011.

Möller T, Burda O, Burmeister S, Heber B, Langner F, Wissmann F. 2012. In-field calibration of the Navigation Dosimetry System (NAVIDOS) during solar minimum conditions. Astrophys Space Sci Trans 8: 45-49. https://doi.org/10.5194/astra-8-45-2012.

Odstrcil D. 2003. Modeling 3-D solar wind structure. Adv Space Res 32(4): 497-506. https://doi.org/10.1016/S0273-1177(03)00332-6.

O'Neill PM. 2010. Badhwar-O'Neill 2010 galactic cosmic ray flux model - revised. IEEE Trans Nucl Sci 57(6): 3148-3153. https://doi.org/10.1109/TNS.2010.2083688.

Owens MJ, Forsyth RJ. 2013. The heliospheric magnetic field. Living Rev Sol Phys 10: 5. https://doi.org/10.12942/lrsp-2013-5.

Pinto RF, Rouillard AP. 2017. A multiple flux-tube solar wind model. Astrophys J 838: 89. https://doi.org/10.3847/1538-4357/ aa6398.

Poedts S. 2018. Forecasting space weather with EUHFORIA in the Virtual Space Weather Modeling Centre. Plasma Phys Control Fusion 61: 014011. https://doi.org/10.1088/1361-6587/aae048.

Poedts S, Kochanov A, Lani A, Scolini C, Verbeke C, et al. 2020. The Virtual Space Weather Modelling Centre. J Space Weather Space Clim 10: A14. https://doi.org/10.1051/swsc/2020012.

Pomoell J, Vainio R. 2012. Influence of solar wind heating formulations on the properties of shocks in the corona. Astrophys J 745: 151. https://doi.org/10.1088/0004-637X/745/2/151.

Pomoell J, Poedts S. 2018. EUHFORIA: European heliospheric forecasting information asset. J Space Weather Space Clim 8: A35. https://doi.org/10.1051/swsc/2018020. 
Pomoell J, Aran A, Jacobs C, Rodríguez-Gasén R, Poedts S, Sanahuja B. 2015. Modelling large solar proton events with the shock-and-particle model. Extraction of the characteristics of the MHD shock front at the cobpoint. J Space Weather Space Clim 5: A12. https://doi.org/10.1051/swsc/2015015.

Pomoell J, Lumme E, Kilpua E. 2019. Time-dependent data-driven modeling of active region evolution using energy-optimized photospheric electric fields. Sol Phys 294: 41. https://doi.org/ 10.1007/s11207-019-1430-x.

Price DJ, Pomoell J, Lumme E, Kilpua EKJ. 2019. Time-dependent data-driven coronal simulations of AR 12673 from emergence to eruption. A\&A 628: A114. https://doi.org/10.1051/0004-6361/ 201935535.

Raeder J, Cramer WD, Germaschewski K, Jensen J. 2017. Using OpenGGCM to compute and separate magnetosphere magnetic perturbations measured on board low earth orbiting satellites. Space Sci Rev 206: 601. https://doi.org/10.1007/s11214-016-0304-x.

Riley P. 2012. On the probability of occurrence of extreme space weather events. Space Weather 10: S02012. https://doi.org/ 10.1029/2011SW000734.

Samara E, Pinto FP, Magdalenić J, Jercic V, Scolini C, Wijsen N, Jebaraj IC, Rodriguez L, Poedts S. 2020. Implementing the MULTI-VP coronal model in EUHFORIA: Results and comparisons with the WSA coronal model. A\&A. Submitted.

Schrijver K, Kauristie K, Aylward A, Denardini CM, Gibson SE, et al. 2015. Understanding space weather to shield society: A global road map for 2015-2025 commissioned by COSPAR and ILWS. Adv Space Res 55(12): 2745-2807. https://doi.org/10.1016/ j.asr.2015.03.023.

Scolini C, Rodriguez L, Mierla M, Pomoell J, Poedts S. 2019. Observation-based modelling of magnetised coronal mass ejections with EUHFORIA. A\&A 626: A122. https://doi.org/10.1051/ 0004-6361/201935053.

Scolini C, Chané E, Temmer M, Kilpua E, Dissauer K, et al. 2020. CME-CME interactions as sources of CME geoeffectiveness: The formation of the complex ejecta and intense geomagnetic storm in 2017 early September. Astrophys J Suppl Ser 247(1): 21. https://doi.org/10.3847/1538-4365/ab6216.

Shiota D, Kataoka R. 2016. Magnetohydrodynamic simulation of interplanetary propagation of multiple coronal mass ejections with internal magnetic flux rope (SUSANOO-CME). Space Weather 14(2): 56-75. https://doi.org/10.1002/2015SW001308.

Skandrani C, Innocenti ME, Bettarini L, Crespon F, Lamouroux J, Lapenta G. 2014. Flip-mhd-based model sensitivity analysis. Nonlinear Process Geophys 21: 539-553. https://doi.org/10.5194/ npg-21-539-2014.
Thomson AWP, McKay AJ, Clarke E, Reay S. 2005. Surface electric fields and geomagnetically induced currents in the Scottish Power grid during the 30 October 2003 geomagnetic storm. Space Weather 3: S11002. https://doi.org/10.1029/2005SW000156.

Tóth G, Sokolov IV, Gombosi TI, Chesney DR, Robert Clauer C, et al. 2005. Space weather modeling framework: A new tool for the space science community. JGR Space Physics 110: A12226. https://doi.org/10.1029/2005JA011126.

Vainio R, Laitinen T. 2007. Monte Carlo simulations of coronal diffusive shock acceleration in self-generated turbulence. Astrophys J 658: 622. https://doi.org/10.1086/510284.

Vainio R, Pönni A, Battarbee M, Koskinen EJ, Afanasiev A, Laitinen T. 2014. A semi-analytical foreshock model for energetic storm particle events inside 1 AU. J Space Weather Space Clim 4: A08. https://doi.org/10.1051/swsc/2014005.

Van der Holst B, Sokolov IV, Meng X, Jin M, Manchester WB IV, Tóth G, Gombosi TI. 2014. Alfvén Wave Solar Model (AWSoM): Coronal heating. Astrophys J 782: 81. https://doi.org/10.1088/ 0004-637X/782/2/81.

Verbeke C, Pomoell J, Poedts S. 2019. The evolution of coronal mass ejections in the inner heliosphere: Implementing the Spheromak model with EUHFORIA. A\&A 627: A111. https://doi.org/10.1051/0004-6361/201834702.

Vršnak B, Magdalenić J, Zlobec P. 2004. Band-splitting of coronal and interplanetary type II bursts. III. Physical conditions in the upper corona and interplanetary space. $A \& A$ 413: 753 . https://doi. org/10.1051/0004-6361:20034060.

Webb DF, Howard TA. 2012. Coronal mass ejections: Observations. Living Rev Sol Phys 9: A3. https://doi.org/10.12942/lrsp-2012-3.

Wijsen N. 2020. PARADISE: A model for energetic particle transport in the solar wind. Dissertation presented in partial fulfilment of the requirements for the degree of Doctor of Science (PhD): Mathematics (KU Leuven) and the degree of Doctor of Physics (Universitat de Barcelona).

Wijsen N, Aran A, Poedts S, Pomoell J. 2019a. Modelling threedimensional transport of solar energetic protons in a corotating interaction region generated with EUHFORIA. A\&A 622: A28. https://doi.org/10.1051/0004-6361/201833958.

Wijsen N, Aran A, Pomoell J, Poedts S. 2019b. The interplanetary spread of solar energetic protons near a high-speed solar wind stream. A\&A 634: A47. https://doi.org/10.1051/0004-6361/ 201935139.

Wintoft P, Wik M, Viljanen A. 2015. Solar wind driven empirical forecast models of the time derivative of the ground magnetic field. J Space Weather Space Clim 5: A7. https://doi.org/10.1051/swsc/ 2015008.

Cite this article as: Poedts S, Lani A, Scolini C, Verbeke C, Wijsen N, et al. 2020. EUropean Heliospheric FORecasting Information Asset 2.0. J. Space Weather Space Clim. 10, 57. https://doi.org/10.1051/swsc/2020055. 